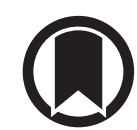

CrossMark

\section{Ventricular mass as a prognostic imaging biomarker in incident pulmonary arterial hypertension}

\author{
To the Editor:
}

Pulmonary arterial hypertension (PAH) is a progressive disease of the pulmonary vasculature that leads to right ventricular (RV) failure and premature death. Because RV failure is the leading cause of mortality in $\mathrm{PAH}$, the prognostic value of cardiac magnetic resonance (CMR) imaging, a powerful tool for assessing the RV, has been the subject of much recent investigation. Several studies have demonstrated that CMR measures of ventricular volumes and right ventricular ejection fraction (RVEF) predict mortality in PAH [1-5]. Fewer studies, however, have examined the prognostic value of ventricular mass metrics in PAH. RAJARAM et al. [5] demonstrated significant associations between RV mass and ventricular mass index (VMI), the ratio of RV mass to left ventricular (LV) mass, and mortality in connective tissue disease-associated pulmonary arterial hypertension (CTD-PAH). LV mass has been shown to predict time to clinical worsening in idiopathic pulmonary arterial hypertension (IPAH) [6]. Small studies have shown that VMI correlates more strongly with invasive haemodynamics than does RV mass alone [7, 8], suggesting added value in a metric incorporating measures of both RV and LV mass.

Between July 2007 and September 2014, we enrolled 89 patients suspected of having PAH with the aim of investigating relationships between ventricular mass and survival in incident PAH cases. All subjects underwent CMR within $48 \mathrm{~h}$ of a right heart catheterisation that defined the presence or absence of PAH. CMR images were acquired and interpreted by a trained radiologist with expertise in cardiac imaging (S.L. Zimmerman) as previously described [9]. Patients found to have PAH were managed in accordance with contemporary guidelines, including prescription of pulmonary vasodilator therapies. Subjects were followed until death or the end of the study period.

CMR metrics were indexed for body surface area, and adjusted for age and sex, as previously described [3]. Relationships between mortality and clinical characteristics, haemodynamics, and CMR metrics were assessed with univariable Cox proportional hazard (CPH) models. CMR variables were scaled to $\log _{1.1}$ for comparison of hazard ratios (HRs). Variables significant in univariable analysis $(\mathrm{p}<0.20)$ and other biologically relevant variables were incorporated into separate bivariable $\mathrm{CPH}$ models. Collinearity was assessed with pairwise correlation and collinear variables were excluded from bivariable models. A p-value $<0.05$ was considered statistically significant.

Of the 89 subjects enrolled, 64 met diagnostic criteria for PAH and were included in the analytic cohort. 42 were classified as having CTD-PAH (with 40 having scleroderma-related PAH) and 22 were judged to have IPAH. Subjects were mean \pm SD $57 \pm 11$ years of age and mostly female (91\%) with CTD-PAH (66\%). The only statistically significant difference in CMR metrics between disease subtypes was a higher mean left ventricular end-diastolic mass index (LVEDMI) in subjects with CTD-PAH $(96 \pm 28 \%$ predicted) compared with IPAH $(79 \pm 17 \%$ predicted $)(\mathrm{p}<0.05)$. Mean RV mass index did not differ significantly in IPAH ( $113 \pm 72 \%$ predicted) versus CTD-PAH ( $121 \pm 69 \%$ predicted). There were 30 deaths ( $46 \%$ mortality) over a median follow-up time of 4.2 years (interquartile range $2.4-5.5$ years).

Unadjusted HRs for mortality are shown in table 1. RV and LV mass metrics, including LVEDMI, LVEDMI \% predicted, right ventricular end-diastolic mass index (RVEDMI), RVEDMI \% predicted and VMI, were all significantly associated with mortality. Each $10 \%$ increase in VMI was associated with $11 \%$

@ERSpublications

Increased right ventricular mass and VMI, the ratio of $\mathrm{RV}$ mass to $\mathrm{LV}$ mass, are predictors of mortality in incident, treatment-naive PAH. These findings challenge the notion that RV hypertrophy is adaptive in pulmonary hypertension. http://ow.ly/qzvt30ntJze

Cite this article as: Simpson CE, Damico RL, Kolb TM, et al. Ventricular mass as a prognostic imaging biomarker in incident pulmonary arterial hypertension. Eur Respir J 2019; 53: 1802067 [https://doi.org/ 10.1183/13993003.02067-2018]. 
TABLE 1 Univariable Cox proportional hazard ratios for mortality for all subjects with pulmonary arterial hypertension

\begin{tabular}{|c|c|c|}
\hline Variable & HR (95\% Cl, p-value) & $\begin{array}{l}\text { HR }(95 \% \mathrm{Cl}, \mathrm{p} \text {-value }) \text { with } \\
\text { variables scaled to } \log _{1.1}\end{array}$ \\
\hline \multicolumn{3}{|l|}{ Clinical characteristics } \\
\hline Age years & $1.02(0.99-1.06,0.17)$ & \\
\hline Female sex & $1.18(0.28-5.01, \mathrm{NS})$ & \\
\hline Caucasian race & $0.21(0.03-1.52,0.12)$ & \\
\hline NYHA FC III-IV & $1.11(0.53-2.36, \mathrm{NS})$ & \\
\hline 6MWD m & $1.00(0.99-1.00,0.08)$ & \\
\hline Presence of CTD & $1.79(0.79-4.05,0.17)$ & \\
\hline \multicolumn{3}{|l|}{ Haemodynamics } \\
\hline mPAP mmHg & $1.01(0.98-1.04, \mathrm{NS})$ & \\
\hline PAWP mmHg & $0.97(0.88-1.07, \mathrm{NS})$ & \\
\hline PVR Wood units & $1.06(1.00-1.13,0.06)$ & \\
\hline $\mathrm{COL} \cdot \mathrm{min}^{-1}$ & $0.72(0.54-0.95,<0.05)$ & \\
\hline Cardiac index $L \cdot \min ^{-1} \cdot \mathrm{m}^{-2}$ & $0.70(0.41-1.19,0.18)$ & \\
\hline \multicolumn{3}{|l|}{ LV metrics } \\
\hline LVESV mL & $0.97(0.94-0.99,<0.05)$ & $0.90(0.83-0.98,<0.05)$ \\
\hline LVEDV mL & $0.98(0.97-0.99,<0.01)$ & $0.88(0.80-0.97,<0.05)$ \\
\hline LVEDVI $\mathrm{mL} \cdot \mathrm{m}^{-2}$ & $0.98(0.96-1.01, \mathrm{NS})$ & $0.95(0.86-1.06, \mathrm{NS})$ \\
\hline LVEDVI \% predicted & $0.98(0.97-1.00,0.17)$ & $0.94(0.85-1.06,0.17)$ \\
\hline LVEDMI $\mathrm{g} \cdot \mathrm{m}^{-2}$ & $1.02(1.001-1.03,<0.05)$ & $1.15(1.02-1.30,<0.05)$ \\
\hline LVEDMI \% predicted & $1.01(1.001-1.03,<0.05)$ & $1.15(1.02-1.30,<0.05)$ \\
\hline \multicolumn{3}{|l|}{ RV metrics } \\
\hline RVEDVI $\mathrm{mL} \cdot \mathrm{m}^{-2}$ & $1.01(0.99-1.02, \mathrm{NS})$ & 1.05 (0.93-1.17, NS) \\
\hline RVEDVI \% predicted & $1.00(0.99-1.01, \mathrm{NS})$ & $1.07(0.95-1.19, \mathrm{NS})$ \\
\hline RVEF \% & $0.98(0.95-1.005,0.11)$ & $0.93(0.85-1.02,0.11)$ \\
\hline RVEF \% predicted & $0.99(0.98-1.01, \mathrm{NS})$ & $0.97(0.88-1.06, \mathrm{NS})$ \\
\hline RVESVI $\mathrm{mL} \cdot \mathrm{m}^{-2}$ & $1.01(1.00-1.02, \mathrm{NS})$ & $1.05(0.98-1.12, \mathrm{NS})$ \\
\hline RVESVI \% predicted & $1.00(0.99-1.00,0.06)$ & $1.06(0.99-1.14,0.06)$ \\
\hline RVEDMI $\mathrm{g} \cdot \mathrm{m}^{-2}$ & $1.02(1.01-1.04,<0.01)$ & $1.11(1.04-1.19,<0.01)$ \\
\hline RVEDMI \% predicted & $1.01(1.003-1.01,<0.01)$ & $1.12(1.05-1.19,<0.01)$ \\
\hline TAPSE mm & $0.93(0.86-0.999,<0.05)$ & $0.93(0.86-1.01,0.07)$ \\
\hline \multicolumn{3}{|l|}{ Composite metrics } \\
\hline VMI & $4.6(1.44-14.7,<0.01)$ & $1.11(1.03-1.19,<0.01)$ \\
\hline SV/ESV & $0.24(0.07-0.80,<0.05)$ & $0.93(0.87-0.99,<0.05)$ \\
\hline SV/PP & $0.65(0.43-1.00,0.05)$ & $0.93(0.87-0.99,<0.05)$ \\
\hline
\end{tabular}

HR: hazard ratio; NYHA FC: New York Heart Association functional class; 6MWD: 6-min walk distance; CTD: connective tissue disease; mPAP: mean pulmonary arterial pressure; PAWP: pulmonary arterial wedge pressure; PVR: pulmonary vascular resistance; CO: cardiac output; LV: left ventricular; LVESV: left ventricular end-systolic volume; LVEDV: left ventricular end-diastolic volume; LVEDVI: left ventricular end-diastolic volume index; LVEDMI: left ventricular end-diastolic mass index; RV: right ventricular; RVEDVI: right ventricular end-diastolic volume index; RVEF: right ventricular ejection fraction; RVESVI: right ventricular end-systolic volume index; RVEDMI: right ventricular end-diastolic mass index; TAPSE: tricuspid annular plane systolic excursion; VMI: ventricular mass index; SV; stroke volume; ESV: end-systolic volume; PP: pulse pressure; NS: not significant $(p>0.20)$.

higher mortality and each 10\% increase in RVEDMI \% predicted was associated with $12 \%$ higher mortality. Each 10\% increase in LVEDMI \% predicted was associated with 15\% higher mortality.

The significance and magnitude of the relationship between RVEDMI \% predicted and mortality persisted in multiple bivariable models adjusting for noncollinear covariates: age, sex, race, 6-min walk distance (6MWD), disease subtype, cardiac index, pulmonary vascular resistance (PVR), LV end-systolic volume, stroke volume (SV)/end-systolic volume (ESV), tricuspid annular plane systolic excursion (TAPSE), and SV/ pulse pressure. Similarly, the significance and magnitude of the relationship between VMI and mortality also persisted with adjustment for noncollinear covariates (age, sex, race, 6MWD, disease subtype, cardiac index, PVR and TAPSE). By contrast, the relationship between LVEDMI \% predicted and mortality was attenuated, and its significance was lost with adjustment for 6MWD, TAPSE and disease subtype.

Our results suggest that RV mass and VMI are candidate prognostic markers in incident PAH. While RV volumes and RVEF were not significantly associated with mortality in the overall cohort, 
associations existed in a survival analysis limited to the IPAH subgroup (data not shown). This finding should be interpreted with caution due to the small size of our cohort, though the pattern aligns with previous work demonstrating differences in the prognostic significance of RV volumetrics in IPAH versus CTD-PAH [3].

Associations between increased RV mass and mortality call into question whether RV hypertrophy is always adaptive in PAH. If instances in which RV hypertrophy may be maladaptive were identified, RV mass metrics might offer earlier prognostic insights than RV volumes or RVEF, which are indicators of dilatation and dysfunction. RV hypertrophy typically occurs earliest in the disease course, then progresses to RV dilatation, dysfunction, and ultimately RV failure and death [10,11]. While RV hypertrophy is generally considered adaptive in PAH, other changes, such as dilatation, are considered maladaptive, with a continuum existing between adaptive and maladaptive change [12]. We noted significant negative correlations between SV/ESV, the noninvasive estimate of RV-pulmonary artery (PA) coupling, and VMI and RVEDMI \% predicted in our cohort $(\mathrm{r}=-0.7, \mathrm{p}<0.01)$. RV-PA coupling reflects the RV ability to adapt to increased afterload in PAH. These negative correlations between measures of coupling and measures of RV hypertrophy provide a basis for speculation that RV hypertrophy may represent maladaptive RV remodelling in some instances.

There may be added value in incorporation of LV metrics into assessments of RV function and adaptation in PAH. Higher LV end-diastolic volumes were associated with decreased risk of mortality in our cohort, a finding also reported by VAN WOLFEREN et al. [1]. It is known that in PAH, the pressure-overloaded right ventricle bows into the left ventricle due to ventricular interdependence imposed by pericardial constraint, thereby underfilling the left ventricle $[13,14]$. This underfilling and unloading of the left ventricle may lead to atrophic LV remodelling over time, which may explain the observed decrease in predicted LV mass in our cohort, with mean LVEDMI 79\% and 96\% predicted in IPAH and CTD-PAH subgroups respectively. As a ratiometric, VMI may thus reflect a degree of RV change further along the continuum toward maladaptive change, when the left ventricle becomes impacted by RV hypertrophy and atrophic LV remodelling occurs.

Our study has several limitations. It was conducted within a single centre, with a modestly sized cohort composed of a high proportion of patients with scleroderma-associated PAH. Thus, these results should be interpreted as hypothesis generating rather than conclusive. Furthermore, there were relatively few patients with IPAH, limiting our power to detect differences between disease subtypes. Our analysis is also limited to associations with baseline CMR metrics only, as few patients within the cohort underwent follow-up CMR. Future studies are needed to examine the prognostic value of mass metrics as possible early markers of maladaptive change in larger cohorts of incident PAH patients with different disease subtypes and to assess the prognostic significance of changes in serially measured mass metrics over time.

Catherine E. Simpson $\oplus^{1,6}$, Rachel L. Damico ${ }^{1,6}$, Todd M. Kolb ${ }^{1}$, Stephen C. Mathai ${ }^{1}$, Rubina M. Khair ${ }^{1}$, Takahiro Sato $^{2}$, Khalil Bourji $\circledast^{3}$, Ryan J. Tedford ${ }^{4}$, Stefan L. Zimmerman ${ }^{5}$ and Paul M. Hassoun ${ }^{1}$

${ }^{1}$ Johns Hopkins University Dept of Medicine, Division of Pulmonary and Critical Care Medicine, Baltimore, MD, USA. ${ }^{2}$ Hokkaido University Hospital First Dept of Medicine, Sapporo, Hokkaido, Japan. ${ }^{3}$ Sinai Hospital of Baltimore Dept of Medicine, Baltimore, MD, USA. ${ }^{4}$ Medical University of South Carolina Dept of Medicine, Division of Cardiology, Charleston, SC, USA. ${ }^{5}$ Johns Hopkins University Dept of Radiology and Radiological Science, Baltimore, MD, USA. ${ }^{6}$ These authors contributed equally to this article.

Correspondence: Paul M. Hassoun, 1830 E. Monument St, Fifth Floor, Baltimore, MD 21205, USA. E-mail: phassou1@ jhmi.edu

Received: Aug 232018 | Accepted after revision: Dec 222018

Author contributions: C.E. Simpson, R.L. Damico and P.M. Hassoun designed the study; R.L. Damico, T.M. Kolb, S.C. Mathai, R.M. Khair and P.M. Hassoun were responsible for patient recruitment, care and follow-up; C.E. Simpson, R.L. Damico, T. Sato, K. Bourji and R.J. Tedford collected, maintained and analysed the data; S.L. Zimmerman interpreted the CMR data; C.E. Simpson and R.L. Damico performed the statistical analyses; C.E. Simpson and R.L. Damico drafted the manuscript; R.L. Damico, R.J. Tedford, S.C. Mathai, T.M. Kolb and P.M. Hassoun critically revised the manuscript for important intellectual content; P.M. Hassoun was principal investigator, had access to all the data in the study, and takes full responsibility for the integrity and accuracy of the data analysis.

Conflict of interest: C.E. Simpson has nothing to disclose. R.L. Damico has nothing to disclose. T.M. Kolb has nothing to disclose. S.C. Mathai reports consultancy fees from Actelion and United Therapeutics, outside the submitted work. R. M. Khair has nothing to disclose. T. Sato has nothing to disclose. K. Bourji has nothing to disclose. R.J. Tedford reports support for a core laboratory from Actelion, outside the submitted work. S.L. Zimmerman has nothing to disclose. P.M. Hassoun has nothing to disclose.

Support statement: This study was supported, in part, by National Institutes of Health (NIH) T32 (NHLBI T32HL007534) (to C.E. Simpson), and NIH P50 HL084946, R01HL114910 and U01HL125175 (to P.M. Hassoun). Funding information for this article has been deposited with the Crossref Funder Registry. 


\section{References}

1 van Wolferen SA, Marcus JT, Boonstra A, et al. Prognostic value of right ventricular mass, volume, and function in idiopathic pulmonary arterial hypertension. Eur Heart J 2007; 28: 1250-1257.

2 van de Veerdonk MC, Kind T, Marcus JT, et al. Progressive right ventricular dysfunction in patients with pulmonary arterial hypertension responding to therapy. J Am Coll Cardiol 2011; 58: 2511-2519.

3 Swift AJ, Capener D, Johns C, et al. Magnetic resonance imaging in the prognostic evaluation of patients with pulmonary arterial hypertension. Am J Respir Crit Care Med 2017; 196: 228-239.

4 Swift AJ, Rajaram S, Campbell MJ, et al. Prognostic value of cardiovascular magnetic resonance imaging measurements corrected for age and sex in idiopathic pulmonary arterial hypertension. Circ Cardiovasc Imaging 2014; 7: 100-106.

5 Rajaram S, Swift AJ, Capener D, et al. Comparison of the diagnostic utility of cardiac magnetic resonance imaging, computed tomography, and echocardiography in assessment of suspected pulmonary arterial hypertension in patients with connective tissue disease. J Rheumatol 2012; 39: 1265-1274.

6 Yamada Y, Okuda S, Kataoka M, et al. Prognostic value of cardiac magnetic resonance imaging for idiopathic pulmonary arterial hypertension before initiating intravenous prostacyclin therapy. Circ J 2012; 76: 1737-1743.

7 Saba TS, Foster J, Cockburn M, et al. Ventricular mass index using magnetic resonance imaging accurately estimates pulmonary artery pressure. Eur Respir J 2002; 20: 1519-1524.

8 Katz J, Whang J, Boxt LM, et al. Estimation of right ventricular mass in normal subjects and in patients with primary pulmonary hypertension by nuclear magnetic resonance imaging. J Am Coll Cardiol 1993; 21: 1475-1481.

9 Kelemen BW, Mathai SC, Tedford RJ, et al. Right ventricular remodeling in idiopathic and scleroderma-associated pulmonary arterial hypertension: two distinct phenotypes. Pulm Circ 2015; 5: 327-334.

10 Haddad F, Doyle R, Murphy DJ, et al. Right ventricular function in cardiovascular disease, part II: pathophysiology, clinical importance, and management of right ventricular failure. Circulation 2008; 117: $1717-1731$.

11 Haddad F, Hunt SA, Rosenthal DN, et al. Right ventricular function in cardiovascular disease, part I: Anatomy, physiology, aging, and functional assessment of the right ventricle. Circulation 2008; 117: 1436-1448.

12 Vonk-Noordegraaf A, Haddad F, Chin KM, et al. Right heart adaptation to pulmonary arterial hypertension: physiology and pathobiology. J Am Coll Cardiol 2013; 62: Suppl., D22-D33.

13 Marcus JT, Gan CT, Zwanenburg JJ, et al. Interventricular mechanical asynchrony in pulmonary arterial hypertension: left-to-right delay in peak shortening is related to right ventricular overload and left ventricular underfilling. J Am Coll Cardiol 2008; 51: 750-757.

14 Vonk-Noordegraaf A, Marcus JT, Gan CT, et al. Interventricular mechanical asynchrony due to right ventricular pressure overload in pulmonary hypertension plays an important role in impaired left ventricular filling. Chest 2005; 128: Suppl., 628S-630S. 